\title{
COMMON FACTORS OF RESULTANTS MODULO $p$
}

\section{DOMINGO GOMEZ, JAIME GUTIERREZ ${ }^{\bowtie}$, ÁLVAR IBEAS and DAVID SEVILLA}

\author{
(Received 16 June 2008)
}

\section{Abstract}

We show that the multiplicity of a prime $p$ as a factor of the resultant of two polynomials with integer coefficients is at least the degree of their greatest common divisor modulo $p$. This answers an open question by Konyagin and Shparlinski.

2000 Mathematics subject classification: primary 13A35; secondary 11C08.

Keywords and phrases: resultant, reduction modulo $p$.

Given two polynomials

$$
F(x)=\sum_{i=0}^{n} \alpha_{i} x^{i} \quad \text { and } \quad G(x)=\sum_{i=0}^{m} \beta_{i} x^{i}
$$

of degree $n$ and $m$ respectively and with integer coefficients, we denote by $S(F, G)$ the Sylvester matrix associated to the polynomials $f$ and $g$, that is,

$$
S(F, G)=\left(\begin{array}{ccccccccc}
\alpha_{0} & & \ldots & & \alpha_{n} & 0 & \ldots & \ldots & 0 \\
0 & \alpha_{0} & & \ldots & & \alpha_{n} & 0 & \ldots & 0 \\
\vdots & & \ddots & & & & \ddots & & \\
0 & \ldots & 0 & \alpha_{0} & & \ldots & & \alpha_{n} & 0 \\
0 & \ldots & \ldots & 0 & \alpha_{0} & & \ldots & & \alpha_{n} \\
\beta_{0} & & \ldots & & \beta_{m} & 0 & \ldots & \ldots & 0 \\
0 & \beta_{0} & & \ldots & & \beta_{m} & 0 & \ldots & 0 \\
\vdots & & \ddots & & & & \ddots & & \\
0 & \ldots & 0 & \beta_{0} & & \ldots & & \beta_{m} & 0 \\
0 & \ldots & \ldots & 0 & \beta_{0} & & \ldots & & \beta_{m}
\end{array}\right) .
$$

We denote by $\operatorname{Res}(F, G)$ the resultant of $F(x)$ and $G(x)$ with respect to $x$, that is,

$$
\operatorname{Res}(F, G)=\operatorname{det} S(F, G),
$$

see $[2,3]$.

This work is partially supported by the Spanish Ministry of Education and Science grant MTM200767088.

(C) 2009 Australian Mathematical Society 0004-9727/2009 \$16.00 
Let $p$ be a prime. It is well known that if the polynomials $F$ and $G$ have a common factor modulo $p$ then $\operatorname{Res}(F, G) \equiv 0 \bmod p$. It is natural to consider the relation between the multiplicity of $p$ as a factor of $\operatorname{Res}(F, G)$ and the degree of this common factor. In some special case, a positive answer to this question has been given in [1, Lemma 5.3] and the problem of extending this result to the general case has been posed in [1, Question 5.4]. Here we give a full solution to this problem.

Let $F G \not \equiv 0 \bmod p$ and let $d_{p}$ be the degree of the gcd of the reductions of $F$ and $G$ modulo $p$. Let $r_{p}$ be the $p$-adic order of $\operatorname{Res}(F, G)$. Then the immediate result is

$$
d_{p}>0 \Rightarrow r_{p}>0 \text {. }
$$

The following theorem is our result.

THEOREM. With the above definitions,

$$
d_{p} \leq r_{p}
$$

PROOF. We shall prove the following result. Let $H(x)$ be a polynomial of degree $t$ such that its leading coefficient is not a multiple of $p$. If $H$ divides $F$ and $G$ modulo $p$, then there exists $\alpha \in \mathbb{Z}$ satisfying

$$
\operatorname{Res}(F, G)=\alpha p^{t} .
$$

By the condition on the leading coefficient of $H$, there exist polynomials

$$
f(x)=\sum_{j=0}^{r} b_{j} x^{j} \quad \text { and } \quad g(x)=\sum_{i=0}^{s} a_{i} x^{i}
$$

with $a_{s} \not \equiv 0 \bmod p, r+t \leq n, s+t \leq m$ and satisfying

$$
F(x) \equiv H(x) f(x) \bmod p, \quad G(x) \equiv H(x) g(x) \bmod p .
$$

We see that

$$
C(x)=F(x) g(x)-G(x) f(x) \equiv 0 \bmod p .
$$

We denote by $R_{i}, i=1, \ldots, m+n$, the row vectors of $S(F, G)$. Recalling that

$$
C(x)=\sum_{i=0}^{s} a_{i} x^{i} F(x)-\sum_{j=0}^{r} b_{j} x^{j} G(x),
$$

we immediately derive that

$$
a_{s} R_{s+1}+\sum_{i=0}^{s-1} a_{i} R_{i+1}-\sum_{j=0}^{r} b_{j} R_{m+j+1} \equiv(0, \ldots, 0) \bmod p .
$$

Similarly, considering $x^{k} C(x)$, we obtain

$$
a_{s} R_{s+k+1}+\sum_{i=0}^{s-1} a_{i} R_{i+k+1}-\sum_{j=0}^{r} b_{j} R_{m+k+j+1} \equiv(0, \ldots, 0) \bmod p,
$$

for $k=0, \ldots, t-1$. 
We consider the matrix $T$ obtained by replacing the rows $R_{s+1}, \ldots, R_{s+t}$ with the rows $a_{s} R_{s+1}, \ldots, a_{s} R_{s+t}$ in $S(F, G)$. Clearly

$$
\operatorname{det} T=a_{s}^{t} \operatorname{det} S(F, G)=a_{s}^{t} \operatorname{Res}(F, G) .
$$

Using (1) we see that, performing elementary row operations on the matrix $T$ that preserve its determinant, we can obtain a certain matrix whose rows $s+1, \ldots, s+t$ are zero vectors modulo $p$. Therefore det $T \equiv 0 \bmod p^{t}$. Recalling that $a_{s} \not \equiv 0 \bmod p$, from (2) we conclude the proof.

The presented proof is also valid for arbitrary unique factorization domains and modulo any principal prime ideal $I=(p)$. In particular, we have the result for any polynomial ring $K\left[x_{1}, \ldots, x_{n}\right][x]$ modulo an irreducible polynomial $p(x) \in$ $K\left[x_{1}, \ldots, x_{n}\right][x]$, where $K$ is an arbitrary field.

On the other hand, the naive generalization of the original result, that is, $d_{p}=r_{p}$, is clearly false as it suffices to choose two polynomials with a common root. We provide an example that shows that the multiplicity can be strictly higher for pairs of polynomials with no common roots.

EXAMPLE 1. The polynomials $x^{2}-2 x$ and $x^{2}-2 x+2$ have no common roots. Let

$$
F(x)=x \cdot\left(x^{2}-2 x\right), \quad G(x)=(x-3)\left(x^{2}-2 x+2\right) .
$$

The greatest common divisor of $F$ and $G$ modulo 3 is $x$. However,

$$
\operatorname{Res}(F, G)=72=3^{2} \cdot 8 .
$$

Finally, the next example shows that $r_{p}-d_{p}$ cannot be bounded even if we bound the degrees of $F$ and $G$.

EXAMPLE 2. For any $p>2$ and any $k>0$, let $F=x(x-1)$ and $G=\left(x-p^{k}\right)$ $(x-2)$. Then $d_{p}=\operatorname{deg} x=1$ and $r_{p} \geq k$.

\section{Acknowledgement}

The authors would like to thank Igor Shparlinski for his interest and for posing this question.

\section{References}

[1] S. V. Konyagin and I. Shparlinski, Character Sums with Exponential Functions and their Applications (Cambridge University Press, Cambridge, 1999).

[2] R. Lidl and H. Niederreiter, Finite Fields (Cambridge University Press, Cambridge, 1997).

[3] B. L. van der Waerden, Modern Algebra (Frederick Ungar Publishing Co., New York, 1964).

DOMINGO GOMEZ, University of Cantabria, E-39071 Santander, Spain e-mail: domingo.gomez@unican.es 
JAIME GUTIERREZ, University of Cantabria, E-39071 Santander, Spain e-mail: jaime.gutierrez@unican.es

ÁLVAR IBEAS, University of Cantabria, E-39071 Santander, Spain e-mail: alvar.ibeas@unican.es

DAVID SEVILLA, Johann Radon Institute for Computational and Applied Mathematics (RICAM), Austrian Academy of Sciences, Altenbergerstraße 69, A-4040 Linz, Austria

e-mail: david.sevilla@oeaw.ac.at 